\title{
CITED1 promotes proliferation of papillary thyroid cancer cells via the regulation of p21 and p27
}

\author{
Hai Li ${ }^{\dagger}$, Hongyu Guan ${ }^{\dagger}$, Yan Guo, Weiwei Liang, Liehua Liu, Xiaoying He, Weijian Ke, Xiaopei Cao, Haipeng Xiao
} and Yanbing $\mathrm{Li}^{*}$

\begin{abstract}
Background: It has been reported that CBP/p300-Interacting Transactivator with glutamic acid [E]/aspartic acid [D]rich C-terminal domain 1 (CITED1) is overexpressed in papillary thyroid cancer (PTC). However, the functional significance and underlying mechanisms of CITED1 in PTC are largely unknown.

Methods: The Cancer Genome Atlas dataset and real-time PCR were used to determine the expression of CITED1 in PTC. The role of CITED1 in PTC cell proliferation was determined conducted using 3-(4,5-dimethylthiazol-2-yl)2,5-diphenyltetrazolium bromide (MTT), colony formation, 5-ethynyl-2'-deoxyuridine (EdU) incorporation, and flow cytometry assays in vitro, and a subcutaneous xenotransplantation tumor model in nude mice was established to analyze tumor growth in vivo. We studied the potential mechanisms underlying the contribution of CITED1 to PTC proliferation using western blotting and luciferase assays.
\end{abstract}

Results: We found that CITED1 was highly expressed in PTC. In vitro and in vivo experiments demonstrated that CITED1 was involved in PTC cell proliferation and tumorigenesis. Then, gain- and loss-of-function experiments revealed that CITED1 decreased the expression of p21 and p27, and thereby increased the phosphorylation of pRb as well as E2F1 transcriptional activity.

Conclusions: Our results suggest that CITED1 is overexpressed in PTC and that CITED1 promotes the proliferation of PTC cells via the regulation of p21 and p27, which indicates that CITED1 might be a potential therapeutic target in the treatment of PTC.

Keywords: CITED1, Papillary thyroid cancer, Proliferation, p21, p27

\section{Introduction}

The incidence of thyroid cancer, which is the most common endocrine malignancy, has continued to increase worldwide over the past several decades $[1,2]$. Thyroid cancer can be classified as follows: follicular epithelial cell-derived papillary thyroid cancer (PTC), follicular thyroid cancer (FTC), and anaplastic thyroid cancer (ATC), and para-follicular C-cell-derived medullary thyroid cancer (MTC). PTC is the most common subtype,

\footnotetext{
*Correspondence: easd04lyb@126.com

${ }^{\dagger} \mathrm{Hai}$ Li and Hongyu Guan contributed equally to this work Department of Endocrinology and Diabetes Center, The First Affiliated Hospital of Sun Yat-sen University, 58 Zhongshan Road II, Guangzhou 510080, Guangdong, China
}

and its standard therapies include surgery, radio-iodine treatment, and thyroid-stimulating hormone suppression [3]. Most patients respond well to these treatments, but some cases are progressive with poor clinical outcomes [4]. Molecular genetic studies have shown a series of genetic and epigenetic changes that are involved in thyroid carcinogenesis $[5,6]$. Further assessment of the genetic events involved in thyroid cancer initiation and progression will provide new insight into thyroid tumorigenesis and may lead to effective therapeutic strategies.

CREB-binding protein/p300 Interacting Transactivator with glutamic acid [E]/aspartic acid [D]-rich C-terminal domain 1 (CITED1) is located on chromosome Xq13.1 and encodes a 27-kDa nuclear protein belonging 
to the CITED family of proteins [7]. This protein family co-regulates transcriptional nuclear proteins via their transactivator domains [7-9]. CITED1 was found to be significantly overexpressed in PTC [10-15]. It has been reported that a lower methylation ratio of CpGs in the promoter region of CITED1 is associated with higher expression of CITED1 mRNA, which suggests the involvement of epigenetic regulation in the overexpression of CITED1 in PTC [16]. Study by Schulten et al. implicated that CITED1 might associated with molecular processes of a brain metastasis from a PTC [17]. In addition, CITED1 might be involved in the roles of some miRNAs in PTC [18].

However, the biologic function and the underlying mechanisms of CITED1 in PTC remain largely unknown.

Here, we investigated the role of CITED1 in the progression of PTC and found that CITED1 can promote the proliferation of PTC cells via the regulation of the expression of $\mathrm{p} 21$ and $\mathrm{p} 27$.

\section{Materials and methods \\ Cell culture}

The human thyroid cancer cell lines TPC1 and BCPAP were provided by Prof. Haixia Guan (China Medical University, China). All cell lines were authenticated by short tandem repeat (STR) DNA profiling and were verified to be mycoplasma-free. The cells were cultured in Dulbecco's modified Eagle's medium (DMEM) (Gibco, Grand Island, NY) supplemented with 10\% fetal bovine serum (FBS; Gibco), $100 \mathrm{U} / \mathrm{mL}$ penicillin (Gibco), and $100 \mathrm{mg} / \mathrm{mL}$ streptomycin (Gibco) at $37{ }^{\circ} \mathrm{C}$ in a $5 \% \mathrm{CO}_{2}$ humidified incubator.

\section{Patients and tissue specimens}

The cases from which the 12 pairs of fresh PTCs and the adjacent nontumorous thyroid tissues were obtained were diagnosed at the First Affiliated Hospital of Sun Yatsen University. The fresh tissues were collected and were then frozen and stored in liquid nitrogen until use. The pathology of all specimens was confirmed by two pathologists. All patients provided informed consent, and ethics approval was obtained from the Institutional Research Ethics Committee.

\section{Vectors and retroviral infection}

The CITED1 construct was generated by sub-cloning PCR-amplified full-length human Clorf106 cDNA into pQCXIP (Clontech, Mountain View, CA). The CITED1shRNAs (TR313896) were purchased from OriGene (Rockville, MD). These plasmids were transfected into PT67 cells (Clontech) using Lipofectamine 3000 (Invitrogen, San Diego, CA). The supernatant was then harvested, passed through a $0.45-\mu \mathrm{m}$ filter and incubated with the indicated cells along with polybrene $(8 \mu \mathrm{g} / \mathrm{mL})$. Subsequently, stable cell lines were selected with $0.5 \mu \mathrm{g} /$ $\mathrm{mL}$ of puromycin for 2 weeks [19].

\section{RNA extraction, RT (reverse transcription), and real-time PCR}

RNA extraction, RT, and real-time PCR were performed as previously described [20]. The primers selected are as follows: CITED1 forward, 5'-GAATCACTCTCT CCTTCTG- $3^{\prime}$ and reverse, 5'-CATCAGCACTTCCTC ATC-3'; glyceraldehyde-3-phosphate dehydrogenase (GAPDH) forward, 5'-TTGAGGTCAATGAAGGGG TC-3' and reverse, 5'-GAAGGTGAAGGTCGGAGT CA- $3^{\prime}$.

\section{Cell viability}

Cell viability was determined using a 3-(4,5-dimethylthiazol-2-yl)-2,5-diphenyltetrazolium bromide (MTT) colorimetric assay. The cells were seeded at a density of $5 \times 10^{3}$ cells per well in 96-well plates. Then, at the same time on days $1,2,3,4,5$ and 6 , the cells were incubated with $20 \mu \mathrm{L}$ MTT (Sigma-Aldrich, St. Louis, MO) per well for $4 \mathrm{~h}$. The culture medium was removed and $200 \mu \mathrm{L}$ dimethyl sulfoxide (DMSO) (Amresco, Solon, $\mathrm{OH})$ was added to each well. The plates were then shaken for $30 \mathrm{~min}$, and the optical density (OD) at $490 \mathrm{~nm}$ was measured using an ELISA plate reader. Each sample was analyzed three times in triplicate.

\section{Flow cytometry analysis}

The indicated cells were harvested and washed in ice-cold phosphate-buffered saline (PBS), which was followed by fixation in $80 \%$ ice-cold ethanol in PBS. After the cells were spun down in a cooled centrifuge and resuspended in cold PBS, RNAase (Sigma-Aldrich) was added at a final concentration of $2 \mu \mathrm{g} / \mathrm{mL}$; the cells were then incubated at $37{ }^{\circ} \mathrm{C}$ for $30 \mathrm{~min}$ followed by incubation in $20 \mu \mathrm{g} / \mathrm{mL}$ of propidium iodide (Sigma-Aldrich) for $20 \mathrm{~min}$ at room temperature. The analysis was performed using a flow cytometer (Beckman-Coulter, Hialeah, FL).

\section{EdU incorporation assay}

The EdU incorporation assay was performed according to the manufacturer's protocol (RiboBio, Guangzhou, China) [21]. Briefly, the indicated cells were cultured in triplicate in 24-well plates for $24 \mathrm{~h}$ and were then treated with $50 \mu \mathrm{M}$ of EdU for $2 \mathrm{~h}$ at $37^{\circ} \mathrm{C}$. After they were fixed in $4 \%$ formaldehyde for $10 \mathrm{~min}$ and permeabilized with $0.5 \%$ Triton X-100 for $10 \mathrm{~min}$ at room temperature, the cells were treated with $1 \times$ Apollo reaction cocktail for $30 \mathrm{~min}$. Subsequently, the cell nuclei were stained with Hoechst 33342 and visualized under a fluorescence 
microscope. Each experiment included three replicates and was performed in triplicate.

\section{Colony formation assay}

For the colony formation assay, cells were plated in 6-well plates at a density of 500 cells per well. The cells were allowed to grow for 10 days at which point they were stained with crystal violet. The plates were imaged, and the numbers of colonies formed by the indicated cells were quantified using the Quantity One software package (Bio-Rad, Hercules, CA). Each experiment was repeated three times.

\section{Western blotting}

Western blotting was performed according to a standard method as previously described [20]. The antibodies used for immunoblotting were as follows: anti-CITED1, anti-cyclin B1, anti-cyclin D3, anti-cyclin A2, anti-cyclin D1, anti-cyclin E1, anti-CDK4, anti-CDK6 (Abcam, Cambridge, MA), anti-cyclin D2, anti-CDK2 (BD Pharmingen, San Diego, CA), anti-cyclin E2, anti-p21Cip1, anti-p27Kip1 (Cell Signaling Technology, Beverly, MA), and anti- $\alpha$-tubulin (Sigma-Aldrich, St. Louis, Missouri). The bands were quantified using Quantity One software (Bio-Rad, Hercules, CA).

\section{In vivo experiments}

Five female BALB/c mice (4 weeks of age) were used to assess the effect of CITED1 on tumor growth in vivo. Briefly, $1 \times 10^{7}$ of the indicated cells were injected subcutaneously into the dorsal flank of each mouse. Tumor size was measured every 5 days, and the tumor volume was estimated. Thirty days after the injection, the mice were euthanized, and the tumors were removed and weighed. All experiments that involved the use of animals were conducted in accordance with the recommendations in the Guide for the Care and Use of Laboratory Animals of the National Institutes of Health. The protocol was approved by the Institutional Animal Care and Use Committee of Sun Yat-sen University.

\section{Reporter assay}

Dual-luciferase reporter assays (Promega, Madison, WI) were performed according to the manufacturer's instructions as previously described [15]. Briefly, the indicated cells were seeded in triplicate in 24-well plates and were allowed to settle for $24 \mathrm{~h}$. The cotransfection of pE2FTA-Luc plasmid (Clontech, San Francisco, CA) and $1 \mathrm{ng}$ pRL-TK Renilla was performed using Lipofectamine 2000 Reagent (Life Technologies, Gaithersburg, MD) according to the manufacturer's protocol. Thirty-six hours after transfection, the cells were harvested and lysed, and the luciferase activity was assessed. The firefly luciferase activity was normalized to that of Renilla luciferase. Three independent experiments were performed.

\section{Statistical analysis}

All data are presented as the means \pm standard deviations (SDs) of at least three independent experiments. Statistical analysis was performed using SPSS17.0 software (SPSS Inc., Chicago, IL), and the Student t-test was used to compare the differences between two groups. $\mathrm{P}<0.05$ was considered significant.

\section{Results}

CITED1 is upregulated in human PTC

Initially, we analyzed the expression of CITED1 in 59 pairs of thyroid tumor specimens and corresponding adjacent noncancerous thyroid tissues using the thyroid cancer RNAseq data deposited in the TCGA. As shown in Fig. 1a, the expression level of CITED1 was significantly elevated in most PTC tissues compared with their paired adjacent noncancerous thyroid tissues. Moreover, CITED1 was significantly upregulated in PTC tissues $(\mathrm{n}=496)$ compared with non-cancerous thyroid tissues $(\mathrm{n}=59)$ in the TCGA cohort (Fig. 1b). Next, we confirmed the expression of CITED1 in the 12 pairs of PTC and adjacent noncancerous thyroid tissues using quantitative RT-PCR. As shown in Fig. 1c, CITED1 expression

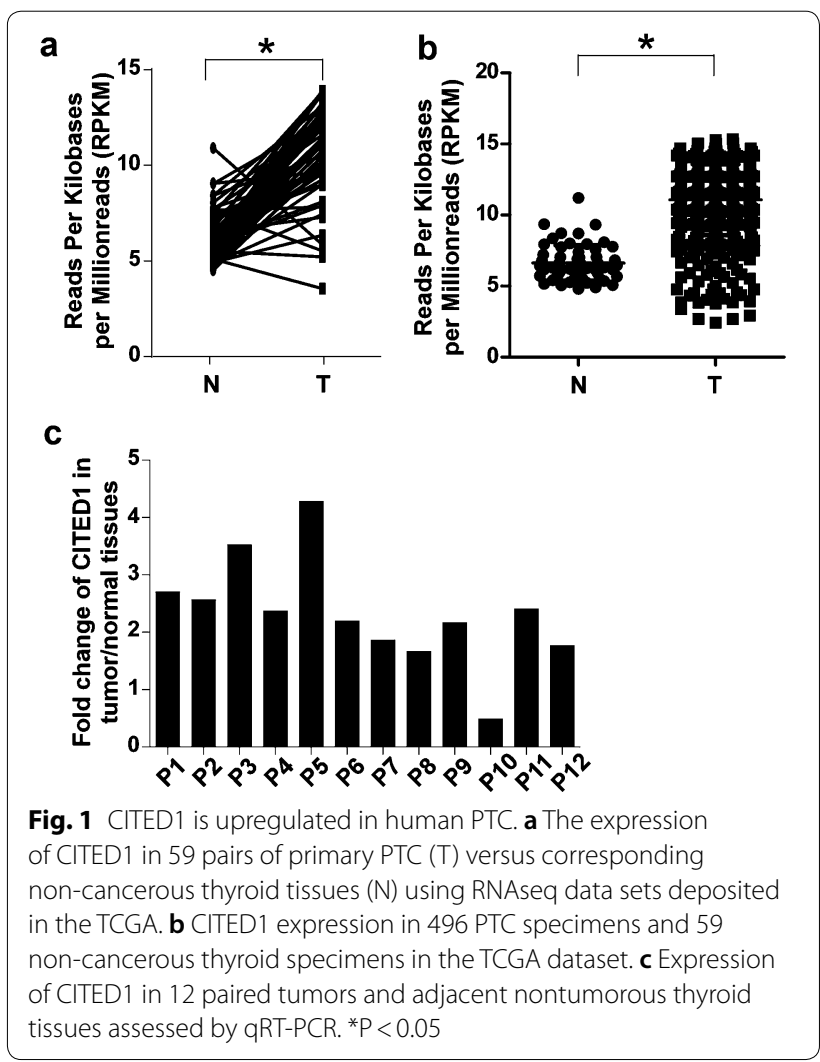


was elevated in tumor tissues compared with corresponding noncancerous tissues.

\section{Silencing of CITED1 inhibits cell proliferation}

To investigate the functional role of CITED1 in PTC progression, TPC1 and BCPAP cells in which CITED1 was stably knocked down were established (Fig. 2a and Additional file 1: Fig. S1a). Next, we evaluated cell viability by MTT assay. As shown in Fig. 2b, the silencing of CITED1 significantly decreased the rate of cell growth compared with control cells. Furthermore, the colony formation ability of the cells after depletion of CITED1 was significantly inhibited compared with that of control cells (Fig. 2c). To investigate the effect of CITED1 on cell cycle progression in PTC cells, fluorescence-activated cell sorting analyses were performed. As shown in Fig. 2d, depletion of CITED1 increased the proportion of cells in G1 phase. Moreover, an EdU incorporation assay was performed, and the results showed that the proportion of EdU-positive cells was significantly decreased in CITED1-silenced cells (Fig. 2e). Taken together, these data demonstrated that the knockdown of CITED1 inhibited PTC cell proliferation.

\section{Ectopic overexpression of CITED1 promotes proliferation of PTC}

To further confirm the role of CITED1 in PTC cell proliferation. TPC1 and BCPAP cells in which CITED1 was overexpressed were established (Fig. 3a and Additional file 1: Fig. S1b). As shown in Fig. 3b, the overexpression of CITED1 significantly increased cell viability compared with the vector-control. Furthermore, the colony formation ability was significantly promoted by CITED1 overexpression (Fig. 3c). As shown in Fig. 3d, flow cytometry analysis revealed that ectopic overexpression of CITED1 increased the proportion of cells in S phase. Moreover, the EdU incorporation assay showed that the proportion of EdU-positive cells was significantly increased in cells with forced expression of CITED1 (Fig. 3e). Taken together, these data demonstrated that ectopic overexpression of CITED1 promoted PTC cell proliferation.

\section{Downregulation of CITED1 suppresses tumor growth in vivo}

To investigate the biological effect of CITED1 silencing on tumor growth in vivo, we proceeded with the establishment of a subcutaneous xenograft tumor model in nude mice. The growth curve revealed a dramatic decrease in tumor growth in the group in which CITED1 was knocked down (Fig. 4a). Moreover, the tumor size (Fig. 4b) and weight (Fig. 4c) of the group in which CITED1 was silenced were lower than the tumor size and weight of the control group. These data further confirmed the role of CITED1 in PTC cell proliferation.

\section{Silencing of CITED1 elevates the expression of the CKIs p21 and $\mathrm{p} 27$}

To delineate the mechanisms underlying the effect of CITED1 on PTC cell proliferation, western blot analysis was performed to assess the protein expression level of cell cycle regulators. As shown in Fig. 5a and Additional file 1: Fig. S1c, the knockdown of CITED1 increased the expression levels of $\mathrm{p} 21$ and $\mathrm{p} 27$ proteins in TPC1 and BCPAP cells, but no changes in the protein levels of cyclin A2, cyclin B1, cyclin D1, cyclin D2, cyclin D3, cyclin $\mathrm{E} 1$, cyclin $\mathrm{E} 2, \mathrm{CDK} 2, \mathrm{CDK} 4$, or CDK6 were observed. We further examined the effects of CITED1 silencing on $\mathrm{pRb}$ phosphorylation and E2F transcriptional activity. As shown in Fig. 5b, c, and Additional file 1: Fig. S1d, depletion of CITED1 decreased the phosphorylation of $\mathrm{pRb}$ and E2F transcriptional activity. Taken together, these results indicated that CITED1 suppressed proliferation through the upregulation of $\mathrm{p} 21$ and $\mathrm{p} 27$.

\section{Overexpression of CITED1 decreases the expression of CKI p21 and p27}

As shown above, the knockdown of CITED1 increased the expression levels of $\mathrm{p} 21$ and $\mathrm{p} 27$ proteins, decreased the phosphorylation of $\mathrm{pRb}$ and inhibited E2F transcriptional activity in TPC1 and BCPAP cells. We further examined the effects of CITED1 overexpression on PTC cells. As shown in Fig. 6a, b and Additional file 1: Fig. S1e, f, ectopic overexpression of CITED1 decreased the expression of $\mathrm{p} 21$ and $\mathrm{p} 27$ proteins, but increased the phosphorylation of $\mathrm{pRb}$ and E2F transcriptional activity. Taken together, these results further suggested that p21 and p27 mediated the effect of CITED1 on PTC cell proliferation.

\section{Discussion}

CITED1 is the central member of the CITED family of transcriptional co-regulators and was originally cloned from a differential display screen between pigmented mouse B16 melanoma cells and their dedifferentiated weakly pigmented derivative, B16F10 cells [7]. Studies have demonstrated that CITED1 is a non-DNA binding nuclear transcriptional co-regulator capable of influencing transcription downstream of the TGF $\beta, E R \alpha$, and Wnt $/ \beta$-Catenin genes. These effects are dependent on the conserved CITED family CR2 domain, which stimulates CITED1-CBP/P300 binding. Moreover, CITED1 is believed to act by stabilizing the CBP/P300-ER $\alpha$ interaction. In some cases, this protein represses the transcription of $\beta$-Catenin by competing with $\mathrm{CBP} / \mathrm{P} 300$ transcriptional co-activators [9, 22-24]. 


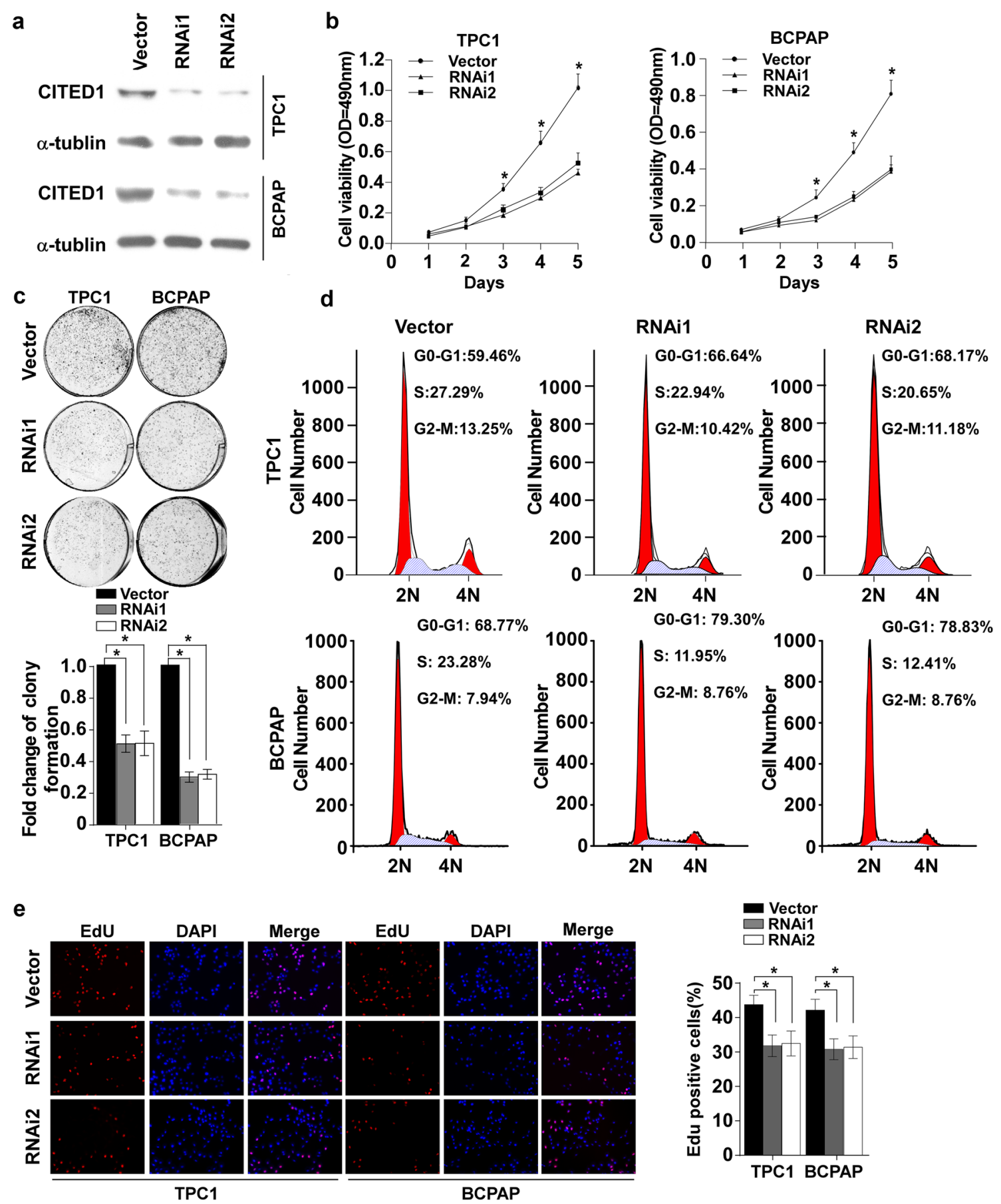

Fig. 2 Silencing of CITED1 inhibits cell proliferation. a Protein expression of CITED1 in the indicated cells was analyzed by western blotting analysis. a-Tubulin was used as the loading control. $\mathbf{b}$ An MTT assay was performed to investigate the effect of CITED1 on the viability of the indicated PTC cells at the indicated time points. c Representative micrographs and relative quantification of colony formation assays of the indicated cells. $\mathbf{d}$ Flow-cytometric determination of the proportion of the studied cells in distinct cell-cycle phases. Knockdown of CITED1 increased the proportion of cells in G1 phase. e Representative images and relative quantification of EdU incorporation assays. For $\mathbf{c}$ and $\mathbf{e}$, the data are reported as the mean \pm SD of three independent experiments. ${ }^{*} \mathrm{P}<0.05$

Notably, accumulating evidence has shown that CITED1 plays critical roles in cancer pathogenesis
[25-30]. Several studies have demonstrated that CITED1 is significantly overexpressed in PTC tissues [10-15]. The 

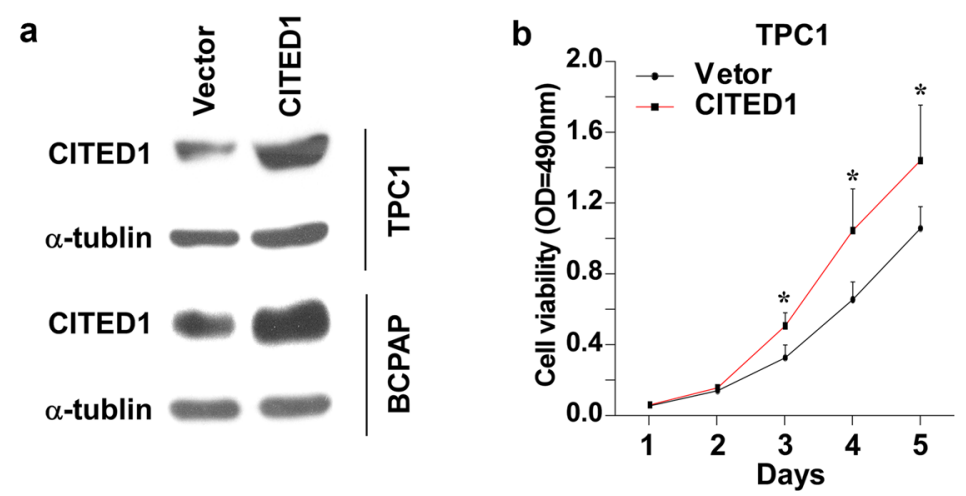

C
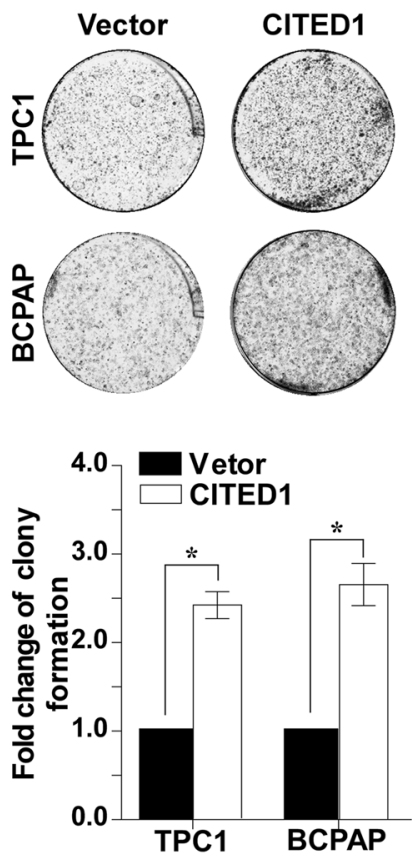

e

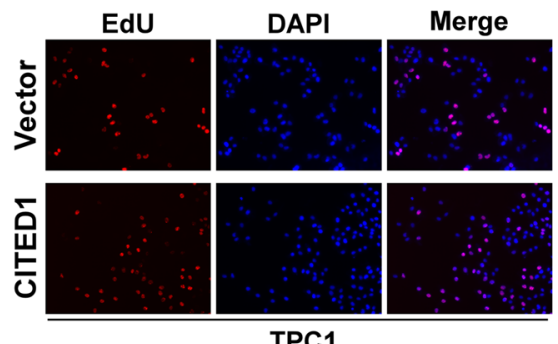

d
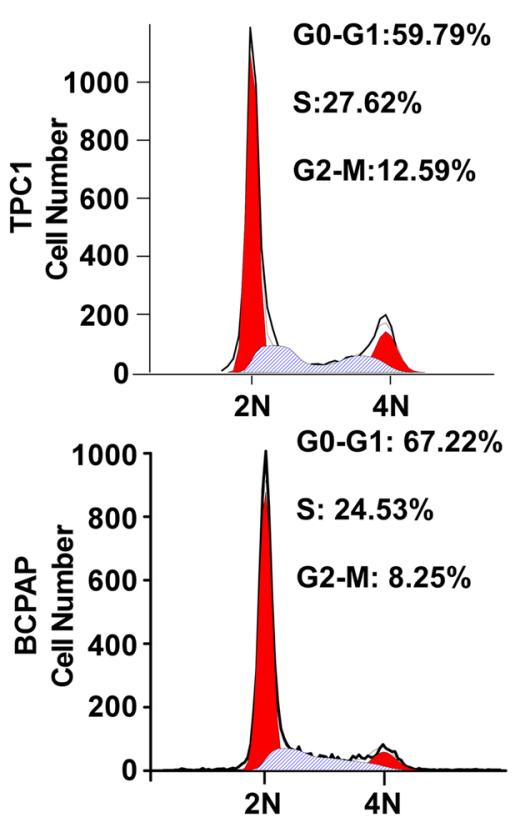

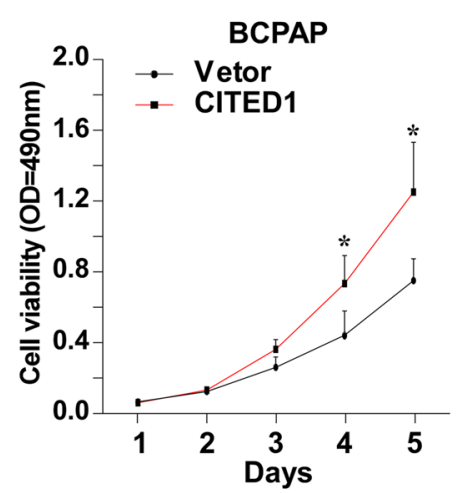

CITED1

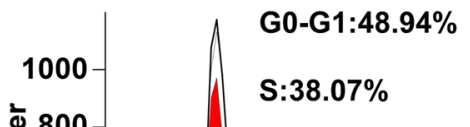

G2-M:12.99\%

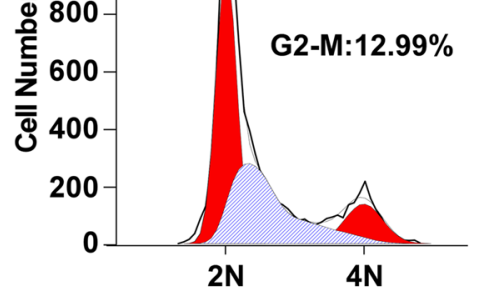

G0-G1: $58.53 \%$

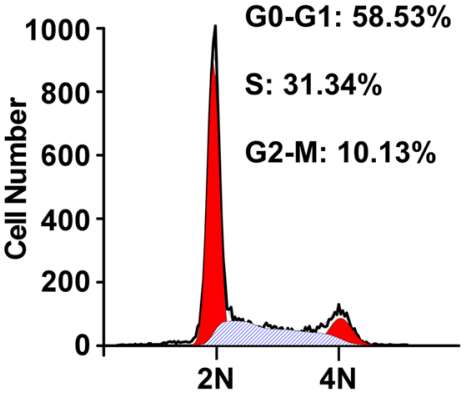

Vetor

CITED1

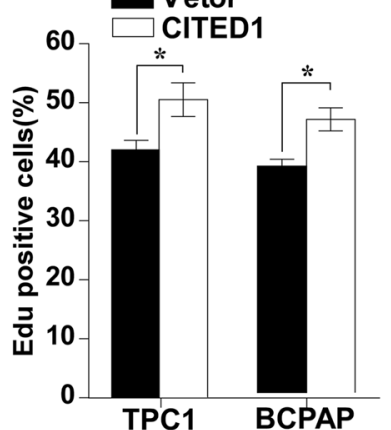

Fig. 3 CITED1 promotes proliferation of PTC cell lines. a Overexpression of CITED1 was confirmed in the studied cells (TPC-1 and BCPAP) by western blotting analysis. a-tubulin was used as the loading control. $\mathbf{b}$ MTT assays were conducted to examine the viability of the indicated cell lines. $\mathbf{C}$ Representative images (upper panel) and relative quantification (lower panel) of the colony formation assay results. $\mathbf{d}$ Proportions of cells in distinct cell-cycle phases in the indicated cell lines were determined by flow cytometry. Overexpression of CITED1 decreased the proprotion of cells in G1 phase. e Representative micrographs (left panel) and quantification (right panel) of the EdU incorporation assay results. For $\mathbf{b}, \mathbf{c}$, and $\mathbf{e}$, the results derived from three independent experiments and are expressed as the mean $\pm S D$. ${ }^{*} P<0.05$ 


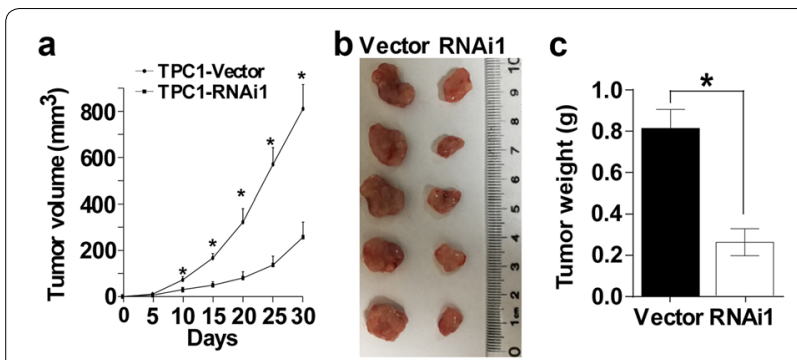

Fig. 4 Downregulation of CITED1 suppresses tumor growth in vivo. a Quantitative analysis of tumor volumes. b Image of subcutaneous tumors isolated from nude mice. c Quantitative analysis of tumor weights. The indicated tumor volumes and weights represent the mean \pm SD of five animals in each group. ${ }^{*} \mathrm{P}<0.05$
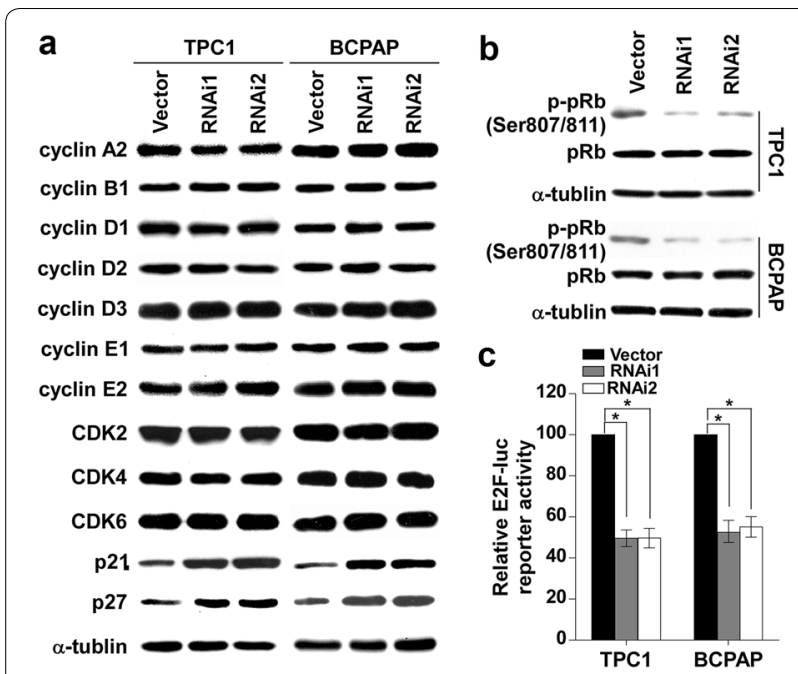

Fig. 5 Knockdown of CITED1 upregulates the expression of the CKIs p21 and p27. a Western blotting analysis was performed to detect the expression levels of the cell cycle regulators cyclin A2, cyclin B1, cyclin D1, cyclin D2, cyclin D3, cyclin E1, cyclin E2, CDK2, CDK4, CDK6, $\mathrm{p} 21^{\mathrm{Cip} 1}$, and $\mathrm{p} 27^{\mathrm{Kip} 1}$ in the indicated cells. a-tubulin was used as the loading control. b Silencing of CITED1 in the studied cells significantly inhibited the phosphorylation of $\mathrm{pRb}$ at the Ser608 residue. a-tubulin served as the sample loading control. c Silencing of CITED1 attenuated E2F transcriptional activity according to the E2F-luc reporter assay. The results are derived from three independent experiments and are expressed as the mean $\pm S D$. ${ }^{*} P<0.05$

results of the current study also showed that CITED1 was overexpressed in clinical PTC specimens, which is consistent with previous studies, and indicated that CITED1 may play an important role in the development and progression of PTC. Indeed, our in vitro and in vivo experiments strongly demonstrated the pro-proliferative role of CITED1 in PTC cells. These results further demonstrated the oncogenic role of CITED1.
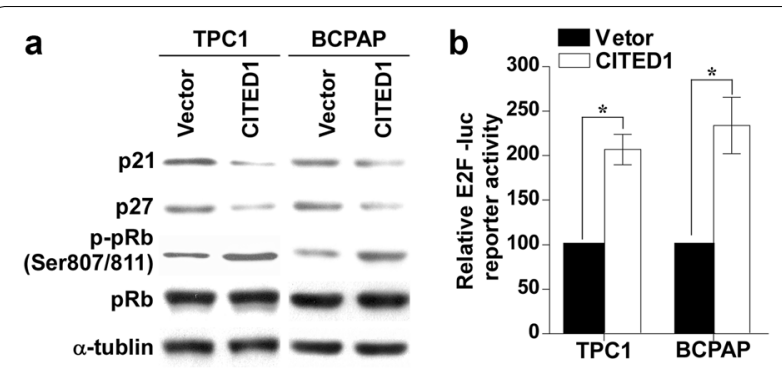

Fig. 6 CITED1 overexpression inhibits the levels of p21 and p27. a Western blotting analysis was performed to detect the expression levels of p21 and p27, as well as the phosphorylation of pRb. a-tubulin was used as the loading control. b Transcriptional activity of E2F was analyzed by luciferase reporter assays. PRL-TK was used to normalize the luciferase activity. For $\mathbf{b}$, data from three independent experiments are shown as the mean \pm SD. ${ }^{*} \mathrm{P}<0.05$

In the additional studies that aimed to delineate the underlying mechanisms of CITED1 in PTC cells, we found that two important cell cycle-related genes, p21 and $\mathrm{p} 27$, were regulated by CITED1. p21 (also known as $p 21^{\text {Wafl/Cip } 1}$, encoded by CDKN1A) is a potent cyclindependent kinase inhibitor that interacts with and inhibits the activity of cyclin-CDK2, -CDK1, and -CDK4/6 complexes. p21 also functions as a regulator of cell cycle progression during $\mathrm{G} 1$ and $\mathrm{S}$ phases. It has been reported that $\mathrm{p} 21$ is the target of the tumor suppressor protein p53 and its isoform [31, 32], and thus functions as a tumor suppressor in a variety of cancer types [33]. p27 (also known as KIP1, encoded by CDKN1B) is an atypical tumor suppressor that regulates $\mathrm{G} 0$ to $\mathrm{S}$ phase transition by binding to and regulating the activity of CDK1 and CDK2. In G0 and early G1, p27 translation and protein stability are maximal, and it binds and inhibits the cyclin E-CDK2 complex [34, 35]. Our data demonstrated that p21 and p27 expression levels were increased and that these proteins were involved in the pro-proliferative effect of CITED1 in PTC cells. These data have provided new insights into the role of CITED1 in the development and progression of malignancies.

In conclusion, these data suggest that CITED1 is overexpressed in PTC tissues. Knockdown of CITED1 inhibits the proliferation of PTC cells, while overexpression of CITED1 promotes the proliferation of PTC cells, and these effects may via regulating p21 and p27. These findings may provide new insights into potential targeted therapies in the treatment of PTC. 


\section{Additional file}

Additional file 1. a-f Quantification of indicated band densities using Quantity One software (Bio-Rad, Hercules, CA).

\section{Abbreviations}

ATC: anaplastic thyroid cancer; CITED1: CBP/p300-InteractingTransactivator with glutamic acid [E]/aspartic acid [D]-rich C-terminal domain 1; DMEM: Dulbecco's Modified Eagle's Medium; FBS: fetal bovine serum; FTC: follicular thyroid cancer; GAPDH: glyceraldehyde-3-phosphate dehydrogenase; MTC: medullary thyroid cancer; PTC: papillary thyroid cancer; RT-PCR: reverse transcription-polymerase chain reaction; TCGA: The Cancer Genome Atlas; WB: western blotting.

\section{Authors' contributions}

HL, HG planned and conducted experiments, analyzed the data and wrote the text. YG, WL, LL, XH, and WK conducted experiments and analyzed data. $\mathrm{XC}$ and $\mathrm{HX}$ participated in its design and contributed to discuss the data and text. YL conceived of the study, and participated in its design and coordination and critical review of the manuscript. All authors read and approved the final manuscript.

\section{Acknowledgements}

We thank Prof. Haixia Guan (China Medical University, China) for TPC-1 and BCPAP cell lines.

\section{Competing interests}

The authors declare that they have no competing interests.

\section{Availability of data and materials}

RNAseqV2 data of 59 pairs of PTC tissues versus paired adjacent noncancerous thyroid tissues and 496 cases of PTC tissues were mined from The Cancer Genome Atlas (TCGA) (https://cancergenome.nih.gov/) using UCSC Xena (http://xena.ucsc.edu/getting-started/)

\section{Consent for publication}

Not applicable.

\section{Ethics approval and consent to participate}

The use of clinical specimens and clinical data were reviewed and approved by the Institutional Research Ethics Committee of the First Affiliated Hospital of Sun Yat-sen University. Written informed consent was obtained from all patients who participated in the study. Animal experiments were approved by the Institutional Animal Care and Use Committee of the First Affiliated Hospital, Sun Yat-sen University.

\section{Funding}

This research was supported by grants from the National Natural Science Foundation of China (Nos. 81572623, 81572624, 81602347) and the Natural Science Foundation of Guangdong Province (No. 2016A030310169).

\section{Publisher's Note}

Springer Nature remains neutral with regard to jurisdictional claims in published maps and institutional affiliations.

Received: 17 June 2018 Accepted: 26 October 2018

Published online: 06 November 2018

\section{References}

1. Vaccarella S, Dal Maso L, Laversanne M, Bray F, Plummer M, Franceschi S. The impact of diagnostic changes on the rise in thyroid cancer incidence: a population-based study in selected high-resource countries. Thyroid. 2015:25(10):1127-36.
2. Cabanillas ME, McFadden DG, Durante C. Seminar thyroid cancer. Lancet. 2016;388(10061):2783-95. https://doi.org/10.1016/S0140-6736(16)30172 $-6$.

3. Brown RL, de Souza JA, Cohen EE. Thyroid cancer: burden of illness and management of disease. J Cancer. 2011;2:193-9.

4. Papewalis C, Ehlers M, Schott M. Advances in cellular therapy for the treatment of thyroid cancer. J Oncol. 2010:2010(4):1-11.

5. Russo D, Damante G, Puxeddu E, Durante C, Filetti S. Epigenetics of thyroid cancer and novel therapeutic targets. J Mol Endocrinol. 2011;46(3):R73-81.

6. Nikiforov YE, Nikiforova MN. Molecular genetics and diagnosis of thyroid cancer. Nat Rev Endocrinol. 2011;7(10):569-80.

7. Shioda T, Fenner MH, Isselbacher KJ. msg1, a novel melanocyte-specific gene, encodes a nuclear protein and is associated with pigmentation. Proc Natl Acad Sci USA. 1996;93(22):12298-303.

8. Fenner MH, Parrish JE, Boyd Y, et al. MSG1 (melanocyte-specific gene 1): mapping to chromosome Xq13.1, genomic organization, and promoter analysis. Genomics. 1998:51(3):401-7.

9. Yahata T, Shao W, Endoh $\mathrm{H}$, et al. Selective coactivation of estrogendependent transcription by CITED1 CBP/p300-binding protein. Genes Dev. 2001;15(19):2598-612

10. Huang Y, Prasad M, Lemon WJ, et al. Gene expression in papillary thyroid carcinoma reveals highly consistent profiles. Proc Natl Acad Sci USA. 2001;98(26):15044-9.

11. Prasad ML, Pellegata NS, Kloos RT, Barbacioru C, Huang Y, de la Chapelle A. CITED1 protein expression suggests papillary thyroid carcinoma in high throughput tissue microarray-based study. Thyroid. 2004;14(3):169-75.

12. Prasad ML, Pellegata NS, Huang Y, Nagaraja HN, de la Chapelle A, Kloos RT. Galectin-3, fibronectin-1, CITED-1, HBME1 and cytokeratin-19 immunohistochemistry is useful for the differential diagnosis of thyroid tumors. Mod Pathol. 2005;18(1):48-57.

13. Aldred MA, Huang Y, Liyanarachchi S, et al. Papillary and follicular thyroid carcinomas show distinctly different microarray expression profiles and can be distinguished by a minimum of five genes. J Clin Oncol. 2004;22(17):3531-9.

14. Scognamiglio T, Hyjek E, Kao J, Chen YT. Diagnostic usefulness of HBME1, galectin-3, CK19, and CITED1 and evaluation of their expression in encapsulated lesions with questionable features of papillary thyroid carcinoma. Am J Clin Pathol. 2006;126(5):700-8

15. Fluge $\varnothing$, Bruland $O$, Akslen LA, Lillehaug JR, Varhaug JE. Gene expression in poorly differentiated papillary thyroid carcinomas. Thyroid. 2006;16(2):161-75.

16. Sassa M, Hayashi Y, Watanabe R, et al. Aberrant promoter methylation in overexpression of CITED1 in papillary thyroid cancer. Thyroid 2011:21(5):511-7

17. Schulten HJ, Hussein D, Al-Adwani F, et al. Microarray expression profiling identifies genes, including cytokines, and biofunctions, as diapedesis, associated with a brain metastasis from a papillary thyroid carcinoma. Am J Cancer Res. 2016;6(10):2140-61.

18. Lu JC, Zhang YP. E2F, HSF2, and miR-26 in thyroid carcinoma: bioinformatic analysis of RNA-sequencing data. Genet Mol Res. 2016:15(1):15017576

19. Kwok WK, Ling M-T, Lee T-W, et al. Up-regulation of TWIST in prostate cancer and its implication as a therapeutic target. Can Res. 2005;65(12):5153-62

20. Guan H, Guo Z, Liang W, et al. Trop2 enhances invasion of thyroid cancer by inducing MMP2 through ERK and JNK pathways. BMC Cancer. 2017;17(1):486

21. Guan H, Liang W, Liu J, et al. Transmembrane protease serine 4 promotes thyroid cancer proliferation via CREB phosphorylation. Thyroid. 2015:25(1):85-94.

22. Shioda T, Lechleider RJ, Dunwoodie SL, et al. Transcriptional activating activity of Smad4: roles of SMAD hetero-oligomerization and enhancement by an associating transactivator. Proc Natl Acad Sci USA. 1998;95(17):9785-90.

23. Yahata T, de Caestecker MP, Lechleider RJ, et al. The MSG1 non-DNAbinding transactivator binds to the p300/CBP coactivators, enhancing 
their functional link to the Smad transcription factors. J Biol Chem 2000;275(12):8825-34

24. Plisov S, Tsang M, Shi G, et al. Cited1 is a bifunctional transcriptional cofactor that regulates early nephronic patterning. J Am Soc Nephrol. 2005;16(6):1632-44.

25. Lovvorn HN III, Westrup J, Opperman S, et al. CITED1 expression in Wilms' tumor and embryonic kidney. Neoplasia. 2007;9(7):589-600

26. Howlin J, Cirenajwis H, Lettiero B, et al. Loss of CITED1, an MITF regulator drives a phenotype switch in vitro and can predict clinical outcome in primary melanoma tumours. PeerJ. 2015;3(7375):e788.

27. Murphy AJ, de Caestecker C, Pierce J, et al. CITED1 expression in liver development and hepatoblastoma. Neoplasia. 2012;14(12):1153-IN23.

28. McBryan J, Howlin J, Kenny PA, Shioda T, Martin F. ERalpha-CITED co-regulated genes expressed during pubertal mammary gland development: implications for breast cancer prognosis. Oncogene. 2007:26(44):6406-19.

29. Nasu T, Oku Y, Takifuji K, et al. Predicting lymph node metastasis in early colorectal cancer using the CITED1 expression. J Surg Res. 2013;185(1):136-42
30. Jiang J, Ma Y, Gao X, Jin Y, Ma X, Yan W, Zhang H, Wang W, Tian T, Zhao Q, Zhang S. Downregulation of Cited 1 suppresses cell proliferation by inducing G2/M arrest of the cell cycle in non-small-cell lung cancer cell lines. Int J Clin Exp Pathol. 2016;9(11):11583-90.

31. Löhr K, Möritz C, Contente A, Dobbelstein M. p21/CDKN1A mediates negative regulation of transcription by p53. J Biol Chem. 2003;278(35):32507-16

32. Rohaly G, Chemnitz J, Dehde S, et al. A novel human p53 isoform is an essential element of the ATR-intra-S phase checkpoint. Cell. 2005;122(1):21-32.

33. Gupta R, Dong Y, Solomon PD, et al. Synergistic tumor suppression by combined inhibition of telomerase and CDKN1A. Proc Natl Acad Sci USA. 2014;111(30):E3062-71.

34. Sherr CJ, Roberts JM. CDK inhibitors: positive and negative regulators of G1-phase progression. Genes Dev. 1999;13(12):1501-12.

35. Chu IM, Hengst L, Slingerland JM. The Cdk inhibitor p27 in human cancer: prognostic potential and relevance to anticancer therapy. Nat Rev Cancer. 2008;8(4):253-67.
Ready to submit your research? Choose BMC and benefit from:

- fast, convenient online submission

- thorough peer review by experienced researchers in your field

- rapid publication on acceptance

- support for research data, including large and complex data types

- gold Open Access which fosters wider collaboration and increased citations

- maximum visibility for your research: over $100 \mathrm{M}$ website views per year

At BMC, research is always in progress.

Learn more biomedcentral.com/submissions 